\title{
IMRT and brachytherapy comparison in gynaecological cancer treatment: thinking over dosimetry and radiobiology
}

\author{
Valentina Pinzi ${ }^{1}$, Valeria Landoni ${ }^{2}$, Federica Cattani ${ }^{3}$, Roberta Lazzari ${ }^{4}$, Barbara Alicja Jereczek-Fossa ${ }^{4,5}$ and Roberto Orecchia ${ }^{6}$ \\ ${ }^{1}$ Department of Neurosurgery, Radiotherapy Unit, Fondazione IRCCS Istituto Neurologico Carlo Besta, 20133 Milan, Italy \\ ${ }^{2}$ Laboratory of Medical Physics and Expert System, IRCCS Istituto Nazionale Tumori Regina Elena, 00128 Rome, Italy \\ ${ }^{3}$ Unit of Medical Physics, European Institute of Oncology IRCCS (IEO), 20141 Milan, Italy \\ ${ }^{4}$ Department of Radiation Oncology of IEO, European Institute of Oncology IRCCS, 20141 Milan, Italy \\ ${ }^{5}$ Department of Oncology and Hemato-Oncology of University of Milan, 20122 Milan, Italy \\ ${ }^{6}$ Scientific Directory of IEO, European Institute of Oncology IRCCS, 20141 Milan, Italy
}

\section{Abstract}

Background: The role of radiotherapy and brachytherapy in the management of locally advanced cervical and endometrial cancer is well established. However, in some cases, intracavitary brachytherapy (ICBRT) is not recommended or cannot be carried out. We aimed to investigate whether external-beam irradiation delivered by means of intensitymodulated radiation therapy (IMRT) might replace ICBRT in gynaecological cancer when the standard ICBRT boost delivering cannot be administered for technical or clinical reasons.

Materials and methods: Fifteen already delivered treatments for gynaecological cancer patients were analysed. The treatments were performed through 3-dimensional conformal radiotherapy (3D-CRT) to the whole-pelvis up to the dose of 45-50.4 Gy followed by a boost dose administered with ICBRT in high-dose-rate or pulsed-dose-rate modality. For each patient, IMRT plans were elaborated to mimic the ICBRT. We analysed the ICBRT boost versus IMRT boost in terms of dosimetric and radiobiological aspects.

Results: Mean conformity index value calculated on boost volume was 0.73 for ICBRT and 0.97 for IMRT. Mean conformation number was 0.24 for ICBRT boost and 0.78 for IMRT boost. Mean normal tissue complication probability (NTCP) values for 3D-CRT plus ICBRT and for IMRT (pelvis plus boost) were, respectively, $28 \%$ and $5 \%$ for rectum; $1.5 \%$ and $0.1 \%$ for urinary bladder and $8.9 \%$ and $6.1 \%$ for bowel.

Conclusions: Our findings suggest that IMRT may represent a viable alternative in delivering the boost in patients diagnosed with gynaecological cancer not amenable to ICBRT.

Key words: cervical cancer, endometrial cancer, brachytherapy, IMRT, radiobiology, dosimetry

Correspondence to: Valentina Pinzi Email: valentinapinzi@yahoo.it and valentina.pinzi@istituto-besta.it

ecancer 2019, 13:993

https://doi.org/10.3332/ecancer.2019.993

Published: 17/12/2019

Received: 18/06/2019

Publication costs for this article were supported by ecancer (UK Charity number 1176307).

Copyright: (c) the authors; licensee ecancermedicalscience. This is an Open Access article distributed under the terms of the Creative Commons Attribution License (http:// creativecommons.org/licenses/by/3.0), which permits unrestricted use, distribution, and reproduction in any medium, provided the original work is properly cited. 


\section{Introduction}

As stated by the International Federation of Gynecology and Obstetrics (FIGO) [1], the role of radiotherapy and brachytherapy in the management of locally advanced cervical and endometrial cancer is well established. According to the clinical and radiological stage, radiation therapy either with or without concurrent chemotherapy followed by brachytherapy [2] is used as exclusive or postoperative treatment. Irradiation is usually delivered by whole-pelvic external beam radiotherapy (EBRT) \pm lomboaortic tract, followed by intracavitary brachytherapy (ICBRT). Selected limited cases can be treated with exclusive pulsed dose rate (PDR) ICBRT. While locally advanced cervical cancer is treated with exclusive EBRT plus ICBRT and chemotherapy, endometrial cancer is generally treated with a surgical approach and the indication to radiotherapy depends on the stage and other histological findings. In some early stages, ICBRT can be the only treatment, while in higher stages, EBRT can be associated. Vaginal vault recurrence in endometrial cancer can be treated with ICBRT alone. In some cases, ICBRT is not recommended or cannot be carried out not only for technical limitations (i.e. difficulty in cannulating the cervix) but also for the rapid fall-off of the dose in challenging scenarios, like bulky disease, irregular geometry of tumour or medical and logistic reasons [3, 4]. Some authors have challenged the use of ICBRT by using EBRT [4-8]. Intensity-modulated radiation therapy (IMRT) can be an alternative approach to deliver a high dose to irregular and concave target volumes while reducing the volume of normal tissues irradiated. Moreover, some studies have already shown that IMRT can substitute ICBRT for boost dose delivery [6-10]. Likewise, the use of IMRT for whole-pelvis irradiation is now widely used and a decreased toxicity to the bowel and other critical structures has been reported with this technique [11-16]. Indeed, IMRT allows performing dose escalation with comparable side effects due to the possibility of a better sparing of organs at risk (OARs) [14, 15, 17].

The aim of this study is to investigate the use of IMRT for the boost treatment as an alternative to ICBRT in terms of dosimetric and radiobiological parameters for gynaecological cancers. We also compared the 3D-CRT plus ICBRT to IMRT pelvis plus boost plans.

\section{Patients and methods}

\section{Patients and treatment characteristics}

The patient population consists of 15 selected patients with histological proven cervical or endometrial cancer, with clinical or pathological stage IB2 bulky-IVA (FIGO stage) [1], treated at the Division of Radiotherapy of the European Institute of Oncology, Milan, Italy, before February 2006. The characteristics of patients are summarised in Table 1.

Clinical and dosimetric data of the patients were retrospectively reviewed.

\section{Treatment planning procedures}

All patients were treated with adjuvant or exclusive whole-pelvis 3D-CRT followed by ICBRT boost.

All patients underwent computed tomography (CT)-based planning with custom immobilisation in the supine position. Clinical target volume (CTV) consisted of tumour volume, also named as gross tumour volume (GTV) plus a margin of 5-7 mm and regional lymph nodes for exclusive treatment, or tumour bed for postoperative treatment. The tumour bed consisted of cervix, uterus, parametria and upper part of the vagina in cervical cancer, vaginal vault in endometrial cancer. The planning target volume (PTV) for the pelvis $($ PTV pelvis $)$ was obtained adding a 7-10 mm margin to the CTV $[9,11,12,18]$. Normal tissues included the rectum wall, urinary bladder wall, bowel and femoral heads.

\section{Current study}

All procedures were in accordance with the ethical standards and with the Helsinki Declaration of 1975. 
Table 1. Patients and baseline disease characteristics.

\begin{tabular}{|l|c|}
\hline \multicolumn{2}{|c|}{ Evaluable patients, $\boldsymbol{n}=15$} \\
\hline Mean age at diagnosis (range), years & $48.4(30-76)$ \\
\hline Tumour Histology & Number (\%) \\
- SCC & $9(60)$ \\
- Adenocarcinoma & $2(13)$ \\
- Endometrioid & $2(13)$ \\
- Mucinous & $1(7)$ \\
- Adenosquamous & $1(7)$ \\
\hline Grading & \\
- G1 & $0(0)$ \\
- G2 & $3(20)$ \\
- G3 & $6(40)$ \\
- NA & $6(40)$ \\
\hline Staging (FIGO) & \\
- IA & $1(7)$ \\
- IB1 & $4(27)$ \\
- IB2 & $5(33)$ \\
- IIA & $1(7)$ \\
- IIB & $3(19)$ \\
- IIIA & $0(0)$ \\
- IIIB & $1(7)$ \\
\hline \multicolumn{2}{|c|}{ Evaluable patients, $\boldsymbol{n}=10$} \\
\hline Pathological T staging & Number (\%) \\
- pT1a & $0(0)$ \\
- pT1b & $4(40)$ \\
- pT2a & $0(0)$ \\
- pT2b & $5(50)$ \\
- Recurrence & $1(10)$ \\
\hline Pathological N staging & $1(10)$ \\
- pNx & $3(30)$ \\
- pN1 & $6(60)$ \\
\hline - & \\
\hline
\end{tabular}

$\mathrm{N}$, nodal; NA, not available; SCC, squamous cell carcinoma;

$\mathrm{T}$, tumour

For the purpose of this study, two treatment plans were prepared for each patient: (a) 3D-CRT plus ICBRT treatment and (b) IMRT (pelvis plus boost) treatment.

(a) 3D-CRT plus ICBRT treatment

The radiation course was administered in two steps: a whole-pelvis 3D-CRT with a four-fields-box technique followed by ICBRT boost. 3D-CRT plans were generated using a commercial treatment planning system (TPS) (Eclipse v.8.6 Varian). The 3D-CRT consisted of four-fields-box plans obtained using $18 \mathrm{MV}$ photons. Anterior, posterior, right lateral and left lateral directions were used.

For the ICBRT boost, patients were equally divided in to three groups, as follows: the first group (I) consisted of five patients who underwent postoperative pelvic 3D-CRT and high-dose-rate (HDR) ICBRT boost (Micro-Selectron HDR; Nucletron Int. B.V., Veenendaal, The Netherlands) using an Ir-192 source Fletcher-Suit-Delclos applicator with intravaginal ovoids. The second group (II) consisted of five 
patients who underwent postoperative pelvic 3D-CRT and PDR ICBRT boost (Micro-Selectron PDR; Nucletron Int. B.V., Veenendaal, The Netherlands) with Fletcher-Suit-Delclos applicator. The third group (III) consisted of five patients who underwent radical pelvic 3D-CRT and PDR ICBRT boost with complete Fletcher-Suit-Delclos applicator with an intrauterine probe. The irradiation boost schedules were as follows: 15 Gy in three fractions for groups I and II, and 15 or $30 \mathrm{~Gy}$ continuously with PDR approach for group III. The dose for each impulse was 0.5 Gy for each PDR treatment.

The ICBRT boost was delivered 2 or 3 weeks after the end of the 3D-CRT. For ICBRT planning, a bladder Foley catheter and a radioopaque rectal probe were used, and a standard Fletcher-Suit-Delclos intracavitary applicator was inserted. A post-implant contrast

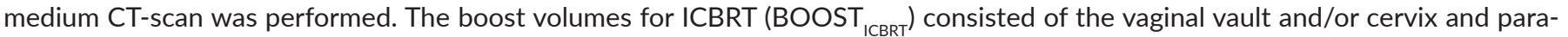
metria plus a 5-mm margin. Rectum wall, urinary bladder wall, bowel and femoral heads were delineated. All volumes were contoured by the same radiation oncologist. An A CT-based 3D dose planning system was used for ICBRT planning (PLATO, Nucletron Int. B.V. Veenendaal, The Netherlands).

(b) IMRT treatment

For the purpose of this study, an IMRT treatment has been planned for each patient.

Each plan was originally optimised and calculated by using an Eclipse (Varian Inc.) TPS. Treatments were simulated with 15-MV linear accelerator equipped with a Millennium multileaf collimator (120 leaves) by a sliding window technique. The dose resolution grid used to calculate the dose distribution was set at $0.5 \times 0.5 \times 0.5 \mathrm{~cm}^{3}$.

The plans included pelvic IMRT followed by sequential IMRT boost. Prescription doses were the same as 3D-CRT for the pelvis, while boost doses were equivalent to the ICBRT doses in terms of biologically effective dose (BED) ( $\alpha / \beta=10 \mathrm{~Gy})$. We contoured on the same CT images already used for the 3D-CRT treatment. The boost volumes consisted of the vaginal vault and/or cervix and parametria plus a 7-10 mm margin for IMRT (BOOST IMRT $_{\text {) }}$ the added margin was larger than the one applied for ICBRT to take into account the set-up errors and organs motion [19, 20].

In Table 2, the OARs and target volumes for the two treatment techniques are reported.

Table 2. OARs and target volumes.

\begin{tabular}{|l|c|c|}
\hline & Volume $\pm \sigma\left(\mathrm{cm}^{3}\right)$ & Range $\left(\mathrm{cm}^{3}\right)$ \\
\hline Target & $1291.29 \pm 184.26$ & $1051.40-1765.96$ \\
\hline PTV pelvis & $37.04 \pm 20.25$ & $11.99-87.60$ \\
\hline ICBRT boost & $94.51 \pm 60.41$ & $36.10-244.70$ \\
\hline IMRT boost & $21.45 \pm 9.11$ & $9.06-48.47$ \\
\hline Rectum wall & $23.42 \pm 10.37$ & $11.80-50.60$ \\
\hline ICBRT boost & $29.86 \pm 8.15$ & $15.30-45.84$ \\
\hline IMRT boost & $31.54 \pm 20.30$ & $9.10-67.40$ \\
\hline Bladder wall &
\end{tabular}

OARs, organs at risk; PTV, planning target volume; ICBRT, intra-cavitary brachytherapy; IMRT, intensity-modulated radiation therapy 


\section{Treatment evaluation}

The PTV coverage, volumes of involved healthy tissue, conformity index (CI) [21] and conformation number (CN) [22], tumour control probabilities (TCP) and normal tissue complication probabilities (NTCP) have been calculated and compared between different radiotherapy approaches.

Prescription doses were established according to the BED calculated with an $\alpha / \beta$ value of $10 \mathrm{~Gy}$.

We compared the boost treatments in terms of dosimetric and radiobiological features. We also evaluated the TCP and NTCP values as complete treatments (3D-CRT plus ICBRT boost versus IMRT pelvis plus boost) in order to test an 'adding dose' method.

\section{Dosimetric evaluations}

We have converted both HDR and PDR ICBRT doses to their biological equivalents at 2 Gy as described in the following radiobiological parameters description. Moreover, the doses delivered by 3D-CRT and ICBRT were added together by using a modified 'parameter adding' method [23]. The dose distribution sum was compared to the one obtained with the IMRT technique.

To evaluate the PTV dose coverage, the percentage of volume receiving $95 \%$ and $85 \%$ of the prescription dose $\left(\mathrm{V}_{95 \%}\right.$ and $\left.\mathrm{V}_{85 \%}\right)$ and the maximum and minimum doses were used. To compare the normal tissue doses, the percentage of rectum and urinary bladder volumes receiving $40 \mathrm{~Gy}\left(\mathrm{~V}_{40 \mathrm{~Gy}}\right)$ and $50 \mathrm{~Gy}\left(\mathrm{~V}_{50 \mathrm{~Gy}}\right)$, the $\mathrm{V}_{40 \mathrm{~Gy}}$ for bowel and $\mathrm{V}_{30 \mathrm{~Gy}}$ for spinal cord were used. Moreover, the value of $\mathrm{D}_{2 \mathrm{~cm}^{3}}$, defined as the minimum dose value in a volume of $2 \mathrm{~cm}^{3}$ receiving the highest dose [23] in the boost treatment, was calculated for urinary bladder and rectum. The doses to the normal tissues were compared in terms of equivalence to 2 Gy per fraction dose with an $\alpha / \beta$ value equal to $3 \mathrm{~Gy}$.

To evaluate the quality of the different treatment approaches, the Radiation Therapy Oncology Group (RTOG) Cl [24] and the CN [25] have been used.

The two parameters were defined as follows:

$$
\begin{gathered}
C l=\frac{T V_{R I}}{T V} \\
C N=\frac{\left(\frac{T V_{R I}}{T V}\right)}{\left(\frac{T V_{R I}}{V_{R I}}\right)}
\end{gathered}
$$

where $T V_{R l}$ is the target volume encompassed by the prescription isodose, $T V$ is the target volume and $V_{R l}$ is the volume encompassed by the prescription isodose; all volumes are expressed in $\mathrm{cm}^{3}$.

$\mathrm{A} \mathrm{Cl}$ value $>1$ indicates that the irradiated volume is greater than the target volume and includes healthy tissues. On the other hand, a $\mathrm{Cl}$ value $<1$ indicates the target volume is only partially irradiated [21].

The value of $\mathrm{CN}$ is comprised between 0 and 1 ; a value of 1 represents a reference isodose covering exactly the target volume without irradiation of healthy tissue and indicates optimal conformation while a value of 0 means no conformation at all.

The $\mathrm{Cl}$ indicator refers to the target itself while CN takes into account both target and healthy tissue irradiation [22].

In Table 3, the $\mathrm{Cl}$ and $\mathrm{CN}$ values are reported. 
Table 3. $\mathrm{Cl}$ and $\mathrm{CN}$ for the different treatments.

\begin{tabular}{|c|c|c|}
\hline & Mean Value $\pm \sigma(\%)$ & Range (\%) \\
\hline \multicolumn{3}{|l|}{$\mathrm{Cl}$} \\
\hline 3D-CRT-pelvis & $0.99 \pm 0.02$ & $0.91-1.00$ \\
\hline IMRT- pelvis & $0.96 \pm 0.02$ & $0.93-0.99$ \\
\hline ICBRT- boost & $0.73 \pm 0.17$ & $0.35-0.91$ \\
\hline IMRT- boost & $0.97 \pm 0.01$ & $0.95-0.99$ \\
\hline \multicolumn{3}{|l|}{$\mathrm{CN}$} \\
\hline 3DCRT- pelvis & $0.47 \pm 0.05$ & $0.39-0.55$ \\
\hline IMRT- pelvis & $0.85 \pm 0.03$ & $0.80-0.88$ \\
\hline ICBRT-boost & $0.24 \pm 0.15$ & $0.03-0.55$ \\
\hline IMRT- boost & $0.78 \pm 0.10$ & $0.62-0.90$ \\
\hline
\end{tabular}

3D-CRT, 3-dimensional conformal radiotherapy; IMRT, intensity-modulated radiation therapy; ICBRT, intra-cavitary brachytherapy

\section{Radiobiological parameters: BED, TCP and NTCP}

According to the literature, IMRT boost prescription doses were established calculating the BED of the ICBRT boost taking into account the dose rate [26-29] as follows:

$$
\mathrm{BED}=D\left\{1+\left(\frac{2 \mathrm{D}}{\mu T \alpha / \beta}\right) *\left[1-(1 / \mu T)\left(1-e^{-\mu T}\right)\right]\right\}
$$

and

$$
\mu=\frac{\ln 2}{T_{1 / 2}}
$$

where $D$ is the total dose, $\alpha$ and $\beta$ are the parameters of the linear-quadratic model and $T_{1 / 2}$ is the half-time for damage repair, being repair rates $\left(T_{1 / 2}\right)$ and $\alpha / \beta$ ratios the main parameters which influence tissue responses when the dose rate is changed $[29,30]$. The value of the parameters $\alpha / \beta$ was set equal to 10 Gy for tumour and 3 Gy for healthy tissue [31] and $T_{1 / 2}$ was 1 hour for tumour and 3 hours for normal tissues [30, 32-36].

In Table 4, biological equivalent boost doses calculated according to eq. (3) for the tumour are reported.

From differential dose-volume histograms (DVHs), TCP and NTCP for rectum, urinary bladder wall and bowel were calculated, according to the Poisson [33] and Lymann-Burman's [37, 38] models, respectively. The used equations were as follows:

$$
\mathrm{TCP}=e^{-\mathrm{N} \cdot e^{-(\alpha+\beta \cdot \theta) \cdot D}}
$$

where $\alpha$ and $\beta$ are the radiobiological parameters, $D$ is the total dose, $d$ is the dose per fraction and $N$ is the clonogenic cell number obtained setting the cell density equal to $10^{5}$ and $10^{7} \mathrm{~cm}^{-3}$ for patients that underwent postoperative and radically radiation treatment, respectively [39].

$$
\mathrm{NTCP}=\frac{1}{\sqrt{2 \pi}} \int_{-\infty}^{x} e^{-\frac{x^{2}}{2}} d x
$$


where $t=\frac{D-T D_{50}(v)}{m \bullet T D_{50}}$ and $T D(v)=T D(1) \bullet v^{-n}$

$v$ is the fractional volume receiving a dose $D, T D$ is the tolerance dose and $T D_{50}$ is the tolerance dose corresponding to a $50 \%$ probability of complications to the healthy tissues, $m$ and $n$ are the parameters of the model. The values of the parameters $m, n$ and $T D_{50}$ were chosen equal to $0.14,0.13$ and $81 \mathrm{~Gy}$, respectively, according to Peeters [40].

Table 5 reports the TCP and NTCP values for rectum, urinary bladder and bowel [41]. For EQD2 calculation, the American Brachytherapy Society worksheet [41] was used (available at https://www.americanbrachytherapy.org/resources/for-professionals/physics-corner/). Tables 6 and 7 summarise the EQD2 values.

\section{Results}

\section{Boost by ICBRT versus IMRT}

As expected, IMRT boost dose distribution was more homogeneous than ICBRT, in fact the comparison was statistically significant in terms of maximum and minimum dosees ( $p=0.001$ and $p<0.0001$, respectively), $\mathrm{V}_{85 \mathrm{~Gy}}$ and $\mathrm{V}_{95 \mathrm{~Gy}}(p=0.0002$ and $p=0.0001$, respectively), and $\mathrm{CN}$ and $\mathrm{Cl}\left(p<0.0001\right.$ and $p=0.0003$, respectively). When comparing OARs doses, the value of $D_{2 \mathrm{~cm}^{3}}$ was in favour of ICBRT. In fact, the $D_{2 \mathrm{~cm}}{ }^{3}$ values were lower for ICBRT than IMRT, being the mean value of the prescription dose for rectum, bowel and urinary bladder of $47.95 \%$, $39.12 \%$ and $30.95 \%$ for ICBRT and $89.54 \%, 91.15 \%$ and $78.57 \%$ for IMRT, respectively.

Table 4. Treatment boost: BED for tumour ( $\alpha / \beta=10 \mathrm{~Gy})$.

\begin{tabular}{|l|l|c|c|}
\hline \multicolumn{2}{|c|}{ ICBRT treatment } & \multicolumn{2}{c|}{ IMRT treatment } \\
\hline HDR 5 Gy $\times 3=15 \mathrm{~Gy}$ & $\mathrm{BED}=22.5 \mathrm{~Gy}$ & $1.8 \mathrm{~Gy} \times 11 \mathrm{fr}=19.8 \mathrm{~Gy}$ & $\mathrm{BED}=23.4 \mathrm{~Gy}$ \\
\hline $\begin{array}{l}\text { PDR 15 Gy } \\
\text { (eq to HDR 4 Gy } \times 3=12 \mathrm{~Gy} \text { ) }\end{array}$ & $\mathrm{BED}=16.8 \mathrm{~Gy}$ & $1.8 \mathrm{~Gy} \times 8 \mathrm{fr}=14.4 \mathrm{~Gy}$ & $\mathrm{BED}=17.0 \mathrm{~Gy}$ \\
\hline $\begin{array}{l}\text { PDR 30 Gy } \\
\text { (eq to HDR 4 Gy } \times 6=24 \mathrm{~Gy} \text { ) }\end{array}$ & $\mathrm{BED}=33.6 \mathrm{~Gy}$ & $1.8 \mathrm{~Gy} \times 16 \mathrm{fr}=28.8 \mathrm{~Gy}$ & $\mathrm{BED}=34.0 \mathrm{~Gy}$ \\
\hline
\end{tabular}

ICBRT, intra-cavitary brachytherapy; IMRT, intensity-modulated radiation therapy; HDR, high dose rate; PDR, pulsed dose rate

Table 5. TCP and NTCP for OARs and target.

\begin{tabular}{|l|c|c|}
\hline & $\begin{array}{c}\text { Mean } \pm \sigma(\%) \\
\text { 3D-CRT plus ICBRT }\end{array}$ & $\begin{array}{c}\text { Mean } \pm \sigma(\%) \\
\text { IMRT }\end{array}$ \\
\hline TCP boost & $80.13 \pm 25.10$ & $99.51 \pm 0.88$ \\
\hline NTCP rectum wall & $27.98 \pm 44.38$ & $4.62 \pm 5.64$ \\
\hline NTCP bladder wall & $1.46 \pm 3.54$ & $0.09 \pm 0.13$ \\
\hline NTCP bowel & $8.95 \pm 7.95$ & $6.09 \pm 3.84$ \\
\hline
\end{tabular}

TCP, tumour control probability; NTCP, normal tissue control probability; 3D-CRT, 3-dimensional conformal radiotherapy; IMRT, intensity-modulated radiation therapy; ICBRT, intra-cavitary brachytherapy 
Table 6. Treatment boost: equivalent biological dose delivered in 2 Gy fractions (EQD2) for tumour ( $\alpha / \beta=10 \mathrm{~Gy}$ ).

\begin{tabular}{|l|c|c|c|}
\hline \multicolumn{2}{|c|}{ ICBRT treatment } & \multicolumn{2}{c|}{ IMRT treatment } \\
\hline HDR 5 Gy $\times 3=15 \mathrm{~Gy}$ & EQD2 $=18.8 \mathrm{~Gy}$ & $1.8 \mathrm{~Gy} \times 11 \mathrm{fr}=19.8 \mathrm{~Gy}$ & EQD2 $=19.5 \mathrm{~Gy}$ \\
\hline $\begin{array}{l}\text { PDR 15 Gy } \\
\text { (eq to HDR 4 Gy } \times 3=12 \mathrm{~Gy} \text { ) }\end{array}$ & EQD2 $=14.2 \mathrm{~Gy}$ & $1.8 \mathrm{~Gy} \times 8 \mathrm{fr}=14.4 \mathrm{~Gy}$ & EQD2 $=14.2 \mathrm{~Gy}$ \\
\hline $\begin{array}{l}\text { PDR 30 Gy } \\
\text { (eq to HDR 4 Gy } \times 6=24 \mathrm{~Gy})\end{array}$ & EQD2 $=28 \mathrm{~Gy}$ & $1.8 \mathrm{~Gy} \times 16 \mathrm{fr}=28.8 \mathrm{~Gy}$ & EQD2 $=28.3 \mathrm{~Gy}$ \\
\hline
\end{tabular}

ICBRT, intra-cavitary brachytherapy; IMRT, intensity-modulated radiation therapy; HDR, high dose rate; PDR, pulsed dose rate

Table 7. EQD2 for tumour $(\alpha / \beta=10 \mathrm{~Gy})$.

\begin{tabular}{|l|c|c|}
\hline & $\begin{array}{c}\text { Mean } \pm \sigma(\%) \\
\text { 3D-CRT plus ICBRT }\end{array}$ & $\begin{array}{c}\text { Mean } \pm \sigma(\%) \\
\text { IMRT }\end{array}$ \\
\hline EQD2 & $70 \pm 2.5$ & $69.9 \pm 4$ \\
\hline
\end{tabular}

EQD2 equivalent biological dose delivered in 2 Gy fractions; 3D-CRT, 3-dimensional conformal radiotherapy; IMRT, intensity-modulated radiation therapy; ICBRT, Intra-cavitary brachytherapy

Analysing TCP and NTCP values, the whole treatment IMRT was relatively superior. However, these results should be taken into account only for the IMRT part, due to the meaningless comparison between 3D-CRT and IMRT for whole pelvis treatment. In fact, the high TCP and low NTCP values of the IMRT analysis can confirm the feasibility of this approach when the ICBRT one is not available. The EQD2 results were similar, by virtue of our study design.

\section{Discussion}

In this study, the radiobiological and dosimetric features of the boost treatment for gynaecological cancer have been analysed: the boost delivered by IMRT was compared to the boost delivered by ICBRT. Even though each technique presents the pros and cons, our results showed that both standard and IMRT approaches theoretically allow treating gynaecological cancer in a safe and effective way. Although it would be interesting to compare the results of postoperative and exclusive settings, the small number of studied patients does not allow a meaningful analysis. Moreover, this study aimed to evaluate only the feasibility of the radiation boost treatment for gynaecological tumours with IMRT in terms of radiobiology and dosimetry and to test a rigorous method for related evaluations. For those reasons, we have chosen a heterogeneous population: both cervical and endometrial patients, post-operative treatments and definitive treatments.

According to Gynaecological Groupe Européen de Curiethérapie-European Society for Therapeutic Radiology and Oncology [(GYN)GECESTRO] working group (II) [42], cumulative DVHs are used for evaluation of the dose heterogeneity.

In gynaecological cancer, the treatment planning and the doses that can be prescribed are significantly influenced by the location of the OARs. Since OARs are close to the brachytherapy sources, only sigmoid bowel instead of the entire bowel is taken into account, besides rectum and urinary bladder. The GTV and CTV in brachytherapy are indeed close to the sources, usually within 15-40 mm, and are dependent on the position of the applicator, size and location of the tumour and cervix. Due to the steep fall-off of the dose close to the sources, there is a significant change in dose and dose-rate throughout the target volumes. The closer to the source, the more pronounced this effect: the dose along an axis perpendicular to the intrauterine source at the level of point A decreases from approximately $200 \%$ to $100 \%$ of the dose to point A when going from 10 to $20 \mathrm{~mm}$ from the source, whereas dose decreases from $100 \%$ to approximately $60 \%$ from 20 to $30 \mathrm{~mm}[42]$. 
Notably, the gradient might be even steeper in terms of biologically equivalent dose, since not only dose but also dose rate follow this gradient effect. This dose inhomogeneity is certainly of major importance for the biological effect of brachytherapy. Therefore, even though IMRT provides a more homogenous dose distribution, this aspect could not be an advantage for tumour control, taking into account that a physical dose delivered by ICBRT could not yield the same biological effects. Moreover, when applying IMRT in place of ICBRT, the crucial key should be the prescription of the dose to the high-risk CTV (CTV-HR) $[43,44]$ as heterogeneous as possible, in order to copycat brachytherapy dosimetry. This could allow delivering more than 80-85Gy EQD2 to CTV-HR [43, 44].

Important to note, when comparing OARs doses, the value of $\mathrm{D}_{2 \mathrm{~cm}^{3}}$ was always lower for ICBRT respect to IMRT. Several investigators [45, 46] compared dosimetric parameters of the entire external OARs volumes with those of only the OARs wall volumes both for urinary bladder and rectum. When the volume of $2 \mathrm{~cm}^{3}$ is considered, the $D_{2 \mathrm{~cm}^{3}}$ values computed for the external contours are almost identical to the $D_{2 \mathrm{~cm}}{ }^{3}$ values for the organ wall. In such cases, this implies that volumes receiving the highest doses are situated entirely within the organ wall. These data resulted in the designation of a new maximal small-volume dose by the gynaecological (GYN)GEC-ESTRO working group for imageguided brachytherapy, where the $D_{2 \mathrm{~cm}^{3}}$ value was designated as the surrogate for a 'hot spot' for OARs [42, 47].

The NTCP calculated for the whole treatment by performing the DVH 'parameter adding' method resulted lower in the case of IMRT for all OARs, thus raising the question whether $D_{2 \mathrm{~cm}^{3}}$ is a representative parameter as a predictor of toxicity [23]. In fact, IMRT will certainly give a very high dose to a small volume of the organ but significant correlation with radiation-induced toxicity have been found mostly with the intermediate-high dose part of the DVH [48].

Interestingly, our predicted results are in agreement with the clinical findings reported by other authors [16] taking into account that the crude rate of incidence of toxicity involves also the effect of chemotherapy; nevertheless, they found that IMRT was associated with low rates of acute and late high-grade toxicity and that outcomes were comparable to those expected with conventional techniques.

Several retrospective cohort studies [4] have provided support for the use of IMRT over conventional techniques but some concern should be considered. First, regarding the potential increasing risk of second malignancies due to the low-dose irradiation of normal tissues [49]. Second, IMRT planning needs to take into consideration both organ motion and tumour regression, as several studies have reported on the inter-fraction and intra-fraction motions of the cervix [50-55]. In fact, Haripotepornkul et al [56] in their study concluded that daily image guidance and possibly re-planning of the treatment volumes are necessary to improve the accuracy of IMRT by accounting for the unpredictable changes in cervical position. Finally, quite large margins that are necessary for IMRT planning might lead to unnecessary overdosing of healthy tissues while the tumour shrinks as a consequence of the therapy. Moreover, uncertainties due to intrafraction variations can contribute to the treatment-related late side [57]. It should be taken into account that as ICBRT necessitates a dedicated brachytherapy unit and costly periodic source changes, IMRT should be applied with a brachytherapy-like immobilisation system and irradiation should be always image-guided delivered. Even though the theory comparing IMRT to ICBRT can demonstrate similar target radiation coverage, the results of clinical studies are certainly more significant. Recently, Holschneider et al [58] published a literature review about brachytherapy for cervical cancer. The authors conclude that the Society of Gynecologic Oncology and the American Brachytherapy Society concur with National Comprehensive Cancer Network (NCCN) guidelines that conformal external beam therapies such as IMRT or stereotactic radiotherapy should not be used as alternatives to brachytherapy in patients undergoing primary curative-intent radiation therapy for cervical cancer [58]. As a matter of fact, the present study has been designed precisely for those patients who cannot be treated with brachytherapy for clinical or personal reasons. Sure enough, IMRT can be exploited to deliver simultaneous integrated boost, thereby reducing the overall treatment time. This overall treatment time reduction might be an advantage not only for the quality of life of the patients but also for increasing tumour control, thus becoming more similar to the low conformed ICBRT boost dose. Moreover, this aspect could increase the treatment tolerance for elderly patients and could reduce the number of older undertreated patients [59].

This study presents some limitations, the most important being heterogeneity and small number of the analysed patients and lack of clinical follow-up data. Furthermore, because we analysed a population of patients treated before 2006, we cannot carry out any comparison to the recent GEC-ESTRO GYN working group paper [60] that defines the current approach to brachytherapy for gynaecological cancers. In fact, advanced EBRT like IMRT has already considered the techniques of choice for these treatments [60].

However, this study defines the pros and cons of advanced EBRT also in place of ICBRT when brachytherapy is unattainable. Both elderly patients and patients with anatomical conformation unsuitable for ICBRT should receive a whole treatment schedule without dose reduction or target missing. 


\section{Conclusions}

More addressed and prospective studies are needed to draw any clear conclusion, though this study confirms that IMRT can provide a valid approach for cervical and endometrial cancer treatment as an alternative to standard radiation therapies when brachytherapy is not feasible.

\section{Conflict of interest}

Nothing to declare.

\section{Funding statement}

This research did not receive any specific grant from funding agencies in the public, commercial, or not-for-profit sectors.

\section{References}

1. FIGO Committee on Gynecologic Oncology (2014) FIGO staging for carcinoma of the vulva, cervix, and corpus uteri Int J Gynaecol Obstet 125(2) 97-98 https://doi.org/10.1016/j.ijgo.2014.02.003 PMID: 24630859

2. Cibula D, Pötter R, and Planchamp F, et al (2018) The European Society of Gynaecological Oncology/European Society for Radiotherapy and Oncology/European Society of Pathology guidelines forthe management of patients with cervical cancer Radiother Oncol 127(3) 404-416 https://doi.org/10.1016/j.radonc.2018.03.003 PMID: 29728273

3. Croke J, Fyles A, and Barbera L, et al (2016) Radiation therapy quality-of-care indicators for locally advanced cervical cancer: a consensus guideline Pract Radiat Oncol 6 315-323 https://doi.org/10.1016/j.prro.2016.01.012 PMID: 27596034

4. O'donnell B, Shiao JC, and Pezzi TA, et al (2018) Stereotactic body radiation therapy, intensity-modulated radiation therapy, and brachytherapy boost modalities in invasive cervical cancer: a study of the National Cancer Data Base Int J Gynecol Cancer 28(3) 563-574 https://doi.org/10.1097/IGC.0000000000001200

5. Mahmoud O, Kilic S, and Khan AJ, et al (2017) External beam techniques to boost cervical cancer when brachytherapy is not an optiontheories and applications Ann Transl Med 5(10) 207 https://doi.org/10.21037/atm.2017.03.102 PMID: 28603722 PMCID: 5451624

6. Neumann O, Kluge A, and Lyubina O, et al (2014) Robotic radiosurgery as an alternative to brachytherapy for cervical cancer patients Strahlenther Onkol 190(6) 538-545 https://doi.org/10.1007/s00066-014-0614-4 PMID: 24589918

7. Cengiz M, Dogan A, and Ozyigit G, et al (2012) Comparison of intracavitary brachytherapy and stereotactic body radiotherapy dose distribution for cervical cancer Brachytherapy 11 125-129 https://doi.org/10.1016/j.brachy.2011.12.001

8. Matsuura K, Okabe T, and Fujita K, et al (2012) Clinical results of external beam radiotherapy alone with a concomitant boost program or with conventional fractionation for cervical cancer patients who did not receive intracavitary brachytherapy $J$ Radiat Res 53(6) 900-905 https://doi.org/10.1093/jrr/rrs051 PMID: 22859563 PMCID: 3483852

9. Feng $\mathrm{CH}$, Hasan $\mathrm{Y}$, and Kopec $\mathrm{M}$, et al (2016) Simultaneously integrated boost (SIB) spares OAR and reduces treatment time in locally advanced cervical cancer J Appl Clin Med Phys 17(5) 76-89 https://doi.org/10.1120/jacmp.v17i5.6123 PMID: 27685108 PMCID: 5874085

10. Hymel R, Jones GC, and Simone CB (2015) Whole pelvic intensity-modulated radiotherapy for gynecological malignancies: a review of the literature Crit Rev Oncol Hematol 94(3) 371-379 https://doi.org/10.1016/j.critrevonc.2014.12.015 PMID: 25600840 PMCID: 4420646 
11. Heron DE, Gerszten K, and Selvaraj RN, et al (2003) Conventional 3D conformal versus intensity-modulated radiotherapy for the adjuvant treatment of gynecologic malignancies: a comparative dosimetric study of dose-volume histograms Gynecol Oncol 91(1) 39-45 https://doi.org/10.1016/S0090-8258(03)00461-X PMID: 14529660

12. Deng X, Han C, and Chen S, et al (2017) Dosimetric benefits of intensity-modulated radiotherapy and volumetric-modulated arc therapy in the treatment of postoperative cervical cancer patients J Appl Clin Med Phys 18(1) 25-31 PMID: 28291936 PMCID: 5689869

13. Kunogi H, Yamaguchi N, and Terao Y, et al (2016) Kidney-sparing methods for extended-field intensity-modulated radiotherapy (EF-IMRT) in cervical carcinoma treatment PLoS One 11(6) e0156623 https://doi.org/10.1371/journal.pone.0156623 PMID: 27258030 PMCID: 4892687

14. Yin G, Wang P, and Lang J, et al (2016) Dosimetric study for cervix carcinoma treatment using intensity modulated radiation therapy (IMRT) compensation based on 3D intracavitary brachytherapy technique J Contemp Brachytherapy 8(3) 221-232 https://doi. org/10.5114/jcb.2016.60590 PMID: 27504132 PMCID: 4965499

15. Lv Y, Wang F, and Yang L, et al (2014) Intensity-modulated whole pelvic radiotherapy provides effective dosimetric outcomes for cervical cancer treatment with lower toxicities Cancer Radiother 18(8) 745-752 https://doi.org/10.1016/j.canrad.2014.08.005 PMID: 25451672

16. Hasselle MD, Rose BS, and Kochanski JD, et al (2011) Clinical outcomes of intensity-modulated pelvic radiation therapy for carcinoma of the cervix Int J Radiat Oncol Biol Phys 80 1436-1445 https://doi.org/10.1016/j.jjrobp.2010.04.041

17. Lan ML, Yu X, and Xiao H, et al (2016) Clinical outcomes and toxicity of postoperative intensity-modulated versus three-dimensional conformal radiation therapy in patients with cervical cancer Asia Pac J Clin Oncol 12(4) 430-436 https://doi.org/10.1111/ajco.12476 PMID: 26923341

18. Taylor A, Rockall A, and Reznek RH, et al (2005) Mapping pelvic lymph nodes: guidelines for delineation in intensity-modulated radiotherapy Int J Radiat Oncol Biol Phys 63 1604-1612 https://doi.org/10.1016/j.ijrobp.2005.05.062 PMID: 16198509

19. International Commission on Radiation Units and Measurements (ICRU) (1985) Dose and volume specification for reporting intracavitary therapy in gynecology: ICRU Report 38 Bethesda: ICRU

20. Lim K, Small W Jr, and Portelance L, et al (2011) Consensus guidelines for delineation of clinical target volume for intensity-modulated pelvic radiotherapy for the definitive treatment of cervix cancer Int J Radiat Oncol Biol Phys 79(2) 348-355 https://doi.org/10.1016/j. ijrobp.2009.10.075

21. Feuvret L, Noel G, and Mazeron JJ, et al (2006) Conformity index: a review Int J Radiat Oncol Biol Phys 64 333-342 https://doi. org/10.1016/j.ijrobp.2005.09.028 PMID: 16414369

22. van't Riet A, Mak AC, and Moerland MA, et al (1997) A conformation number to quantify the degree of conformality in brachytherapy and external beam irradiation: application to the prostate Int J Radiat Oncol Biol Phys 37 731-736 https://doi.org/10.1016/S03603016(96)00601-3

23. van De Kamer JB, De Leeuw AA, and Moerland MA, et al (2010) Determining DVH parameters for combined external beam and brachytherapy treatment: 3D biological dose adding for patients with cervical cancer Radiother Oncol 94 248-253 https://doi.org/10.1016/j. radonc.2009.12.011 PMID: 20080309

24. Lomax NJ and Scheib SG (2003) Quantifying the degree of conformity in radiosurgery treatment planning Int J Radiat Oncol Biol Phys 55 1409-1419 https://doi.org/10.1016/S0360-3016(02)04599-6 PMID: 12654454

25. International Commission on Radiation Units and Measurements (ICRU) (1993) Prescribing, recording and reporting photon beam therapy: ICRU Report 50 Bethesda: ICRU

26. Nag S and Gupta N (2000) A simple method of obtaining equivalent doses for use in HDR brachytherapy Int J Radiat Oncol Biol Phys 46 507-513 https://doi.org/10.1016/S0360-3016(99)00330-2 PMID: 10661360 
27. Sminia P, Schneider CJ, and Koedooder K, et al (1998) Pulse frequency in pulsed brachytherapy based on tissue repair kinetics Int J Radiat Oncol Biol Phys 41 139-150 https://doi.org/10.1016/S0360-3016(98)00023-6 PMID: 9588929

28. Fowler JF (2010) 21 years of biologically effective dose Br J Radiol 83 554-568 https://doi.org/10.1259/bjr/31372149 PMID: 20603408 PMCID: 3473681

29. Dale RG (1985) The application of the linear-quadratic dose-effect equation to fractionated and protracted radiotherapy $\mathrm{Br} J \mathrm{Radio} 58$ 515-528 https://doi.org/10.1259/0007-1285-58-690-515 PMID: 4063711

30. Dale RG and Jones B (1998) The clinical radiobiology of brachytherapy Br J Radiol 71 465-483 https://doi.org/10.1259/bjr.71.845.9691890 PMID: 9691890

31. Boyle J, Craciunescu O, and Steffey B, et al (2014) Methods, safety, and early clinical outcomes of dose escalation using simultaneous integratedand sequential boosts in patients with locally advanced gynecologic malignancies Gynecol Oncol 135(2) 239-243 https://doi. org/10.1016/j.ygyno.2014.08.037 PMID: 25192879

32. Visser AG, Van Den Aardweg GJ, and Levendag PC (1996) Pulsed dose rate and fractionated high dose rate brachytherapy: choice of brachytherapy schedules to replace low dose rate treatments Int J Radiat Oncol Biol Phys 34 497-505 https://doi.org/10.1016/03603016(95)02054-3 PMID: 8567354

33. Brenner DJ and Hall EJ (1991) Conditions for the equivalence of continuous to pulsed low dose rate brachytherapy Int J Radiat Oncol Biol Phys 20 181-190 https://doi.org/10.1016/0360-3016(91)90158-Z PMID: 1993627

34. Millar WT, Hendry JH, and Canney PA (1996) The influence of the number of fractions and bi-exponential repair kinetics on biological equivalence in pulsed brachytherapy BrJ Radiol 69 457-468 https://doi.org/10.1259/0007-1285-69-821-457 PMID: 8705185

35. Galelli M and Feroldi P (1993) The time variable dose-rate in HDR stepping source brachytherapy Radiother Oncol 27 173-174 https:// doi.org/10.1016/0167-8140(93)90141-T PMID: 8356231

36. Warkentin B, Stavrev P, and Stavreva N, et al (2004) A TCP-NTCP estimation module using DVHs and known radiobiological models and parameters sets J Appl Clin Med Phys 5 50-63 https://doi.org/10.1120/jacmp.v5i1.1970

37. Kutcher GJ, Burman C, and Brewster L, et al (1991) Histogram reduction method for calculating complication probabilities for threedimensional treatment planning evaluations Int J Radiat Oncol Biol Phys 21(1) 137-146 https://doi.org/10.1016/0360-3016(91)90173-2 PMID: 2032884

38. Lyman JT and Wolbarst $A B$ (1987) Optimization of radiation therapy, III: a method of assessing complication probabilities from dosevolume histograms Int J Radiat Oncol Biol Phys 13 103-109 https://doi.org/10.1016/0360-3016(87)90266-5 PMID: 3804804

39. Wyatt RM, Beddoe AH, and Dale RG (2003) The effects of delays in radiotherapy treatment on tumour control Phys Med Biol 48 139-155 https://doi.org/10.1088/0031-9155/48/2/301 PMID: 12587901

40. Peeters ST, Hoogeman MS, and Heemsbergen WD, et al (2006) Rectal bleeding, fecal incontinence, and high stool frequency after conformal radiotherapy for prostate cancer: normal tissue complication probability modeling Int J Radiat Oncol Biol Phys 66 11-19 https:// doi.org/10.1016/j.ijrobp.2006.03.034 PMID: 16757129

41. Viswanathan AN, Beriwal S, and De Los Santos JF, et al (2012) American Brachytherapy Society consensus guidelines for locally advanced carcinoma of the cervix. Part II: high-dose-rate brachytherapy Brachytherapy 11 47-52 https://doi.org/10.1016/j.brachy.2011.07.002 PMID: 22265437 PMCID: 3489267

42. Pötter R, Haie-Meder C, and Van Limbergen E, et al (2006) Recommendations from gynaecological (GYN) GEC-ESTRO working group (II): concepts and terms in 3D image-based treatment planning in cervix cancer brachytherapy-3D dose volume parameters and aspects of 3D image-based anatomy, radiation physics, radiobiology Radiother Oncol 78 67-77 https://doi.org/10.1016/j.radonc.2005.11.014 
43. Romano KD, Hill C, and Trifiletti DM, et al (2018) High dose-rate tandem and ovoid brachytherapy in cervical cancer: dosimetric predictors of adverse events Radiat Oncol 13(1) 129 https://doi.org/10.1186/s13014-018-1074-2 PMID: 30012164 PMCID: 6048838

44. Lowrey N, Nilsson S, and Moutrie Z, et al (2015) Comparison of CT-based volumetric dosimetry with traditional prescription points in the treatment of cervical cancer with PDR brachytherapy J Med Imag Radiat Oncol 59(5) 640-645 https://doi.org/10.1111/17549485.12341

45. Siavashpour Z, Aghamiri MR, and Jaberi R, et al (2016) A comparison of organs at risk doses in GYN intracavitary brachytherapy for different tandem lengths and bladder volumes J Appl Clin Med Phys 17(3) 5-13 https://doi.org/10.1120/jacmp.v17i3.5584 PMID: 27167253 PMCID: 5690927

46. Shwetha B, Ravikumar M, and Palled SR, et al (2011) Dosimetric comparison of high dose rate brachytherapy and intensity-modulated radiation therapy for cervical carcinoma J Med Phys 36(2) 111-116 https://doi.org/10.4103/0971-6203.79687 PMID: 21731228 PMCID: 3119952

47. Haie-Meder C, Pötter R, and Van Limbergen E, et al (2005) Recommendations from gynaecological (GYN) GEC-ESTRO working group (I): concepts and terms in 3D image based 3D treatment planning in cervix cancer brachyterapy with emphasis on MRI assessment of GTV and CTV Radiother Oncol 74 235-245 https://doi.org/10.1016/j.radonc.2004.12.015 PMID: 15763303

48. Michalski JM, Gay H, and Jackson A, et al(2010) Radiation dose-volume effects in radiation-induced rectal injury Int J Radiat Oncol Biol Phys 76 S123-S129 https://doi.org/10.1016/j.ijrobp.2009.03.078 PMID: 20171506 PMCID: 3319467

49. Zwahlen DR, Ruben JD, and Jones P, et al (2009) Effect of intensity-modulated pelvic radiotherapy on second cancer risk in the postoperative treatment of endometrial and cervical cancer Int J Radiat Oncol Biol Phys 74 539-545 https://doi.org/10.1016/j.ijrobp.2009.01.051 PMID: 19427555

50. Maemoto H, Toita T, and Ariga T, et al (2017) Predictive factors of uterine movement during definitive radiotherapy for cervical cancer J Radiat Res 58(3) 397-404 PMCID: 5441382

51. Seppenwoolde $Y$, Stock M, and Buschmann M, et al (2016) Impact of organ shape variations on margin concepts for cervix cancer ART Radiother Oncol 120(3) 526-531 https://doi.org/10.1016/j.radonc.2016.08.004 PMID: 27528119

52. Li XA, Qi XS, and Pitterle M, et al (2007) Interfractional variations in patient setup and anatomic change assessed by daily computed tomography Int J Radiat Ocol Biol Phys 68 581-591 https://doi.org/10.1016/j.ijrobp.2006.12.024

53. Belfatto A, Riboldi $M$, and Ciardo D, et al (2016) Adaptive mathematical model of tumor response to radiotherapy based on CBCT data IEEE J Biomed Health Inform 20(3) 802-809 https://doi.org/10.1109/JBHI.2015.2453437

54. Belfatto A, Riboldi M, and Ciardo D, et al (2016) Kinetic models for predicting cervical cancer response to radiation therapy on individual basis using tumor regression measured in vivo with volumetric imaging Technol Cancer Res Treat 15(1) 146-158 https://doi. org/10.1177/1533034615573796

55. Belfatto A, Riboldi M, and Ciardo D, et al (2016) Modeling the interplay between tumor volume regresione and oxygenation in uterine cervical cancer during radiotherapy treatment. IEEE J Biomed Health Inform 20(2) 596-605 https://doi.org/10.1109/JBHI.2015.2398512

56. Haripotepornkul NH, Nath SK, and Scanderbeg D, et al (2011) Evaluation of intra- and inter-fraction movement of the cervix during intensity modulated radiation therapy Radiother Oncol 98 347-351 https://doi.org/10.1016/j.radonc.2010.11.015 PMID: 21216480

57. Lakosi F, De Cuypere M, and Viet Nguyen P, et al (2015) Clinical efficacy and toxicity of radiochemotherapy and magnetic resonance imaging-guided brachytherapy for locally advanced cervical cancer patients: a mono-institutional experience Acta Oncol 54(9) 15581566 https://doi.org/10.3109/0284186X.2015.1062542 PMID: 26406152 
58. Holschneider CH, Petereit DG, and Chu C, et al (2019) Brachytherapy: a criticalc component of primary radiation therapy for cervical cancer: from the Society of Gynecologic Oncology (SGO) and the American Brachytherapy Society (ABS) Brachytherapy 18(2) 123-132 https://doi.org/10.1016/j.brachy.2018.11.009 PMID: 30665713

59. Pinzi V and Trovò M (2014) Postoperative radiotherapy for elderly endometrial cancer patients: worsening frailty or lifesaving measure? World Cancer Res J 1(2) e209

60. Potter R, Tanderup K, and Kiristis C, et al (2019) The EMBRACE II study: the outcome and prospect of two decades of evolution within the GEC-ESTRO GYN working group and the EMBRACE studies Clin Transl Radiat Oncol 9 48-60 https://doi.org/10.1016/j. ctro.2018.01.001 